(c) 2010 IEEE. Personal use of this material is permitted. Permission from IEEE must be obtained for all other uses, in any current or future media, including reprinting/republishing this material for advertising or promotional purposes, creating new collective works, for resale or redistribution to servers or lists, or reuse of any copyrighted component of this work in other works. 


\section{THE ROLE OF WIRELESS SENSOR NETWORKS (WSNs) IN INDUSTRIAL OIL AND GAS CONDITION MONITORING}

\author{
Mohammad reza Akhondi, Alex \\ Talevski \\ DEBII \\ Curtin University of Technology, \\ Perth, Western Australia \\ M.Akhondi@postgrad.curtin.edu.au \\ A.Talevski@curtin.edu.au
}

\author{
Simon Carlsen \\ Statoil ASA, Trondheim, \\ Norway \\ SCAR@StatoilHydro.com
}

\author{
Stig Petersen \\ SINTEF ICT, Trondheim, \\ Norway \\ stig.petersen@ sintef.no
}

\begin{abstract}
Wireless sensor networks have a vast amount of applications including environmental monitoring, military, ecology, agriculture, inventory control, robotics and health care. This paper focuses on the area of monitoring and protection of oil and gas operations using wireless sensor networks that are optimized to decrease installation, and maintenance cost, energy requirements, increase reliability and improve communication efficiency. In addition, simulation experiments using the proposed model are presented. Such models could provide new tools for research in predictive maintenance and condition-based monitoring of factory machinery in general and for "open architecture machining systems" in particular. Wireless sensing no longer needs to be relegated to locations where access is difficult or where cabling is not practical. Wireless condition monitoring systems can be cost effectively implemented in extensive applications that were historically handled by running routes with data collectors. The result would be a lower cost program with more frequent data collection, increased safety, and lower spare parts inventories. Facilities would be able to run leaner because they will have more confidence in their ability to avoid downtime.
\end{abstract}

Keywords: Ad hoc and sensor networks, routing, addressing schemes, wireless networks.

\section{INTRODUCTION}

The oil and gas industry includes processes for exploration, extraction, refining, transporting, and marketing petroleum products. The largest volume products of the industry are fuel, oil and gasoline (petrol). Petroleum is also the raw material for many chemical products, including pharmaceuticals, solvents, fertilizers, pesticides, and plastics. As the demand for fossil fuels continues to grow, oil and gas companies will have to develop new technologies and improve operations in order to increase productivity and expand on their current abilities.

Research in the field of Wireless Sensor Networks that are applied in condition monitoring is relatively active. Current investigations mostly focus on efficient routing protocols for ad hoc and wireless sensor networks [1], quality of service (QoS) support [2] [3], security [4], and middleware [5]. Most of these issues are investigated under the assumption that the network used for sensors does not have a predetermined infrastructure. Fortunately, the wireless sensor network in oil and gas industries especially in pipeline applications is a structured network in which sensor nodes are physically distributed in a straight line. This characteristic can be utilized for enhancing the communication quality and reliability in the pipeline systems. This paper addresses the issues and challenges of using wireless sensor networks for monitoring and protection of oil and gas operations. Also, it presents a routing protocol and addressing scheme for these special kinds of sensor networks. This architecture utilizes the special linear structure of the networks to solve some of communication reliability and security problems. The objective of the design is to reduce installation and maintenance costs, increase network reliability and fault tolerance, increase battery life for wireless sensors, reduce end-to-end communication delay for QoS sensitive data, and increase network lifetime.

\section{ADVANTAGES OF CONDITION MONITORING BY WIRELESS NETWORK SENSORS}

\section{A. Cost justification}

The key to the widespread use of condition monitoring sensors is low cost: both installation and operating costs. Data collectors are considered by many to be the lowest cost method for monitoring vibration. With monthly or quarterly route running, companies can prevent many failures and provide an acceptable cost justification to management. While the installed costs of data collectors are typically the lowest, operating costs are higher due to continuing labor expense. When analyzed over a period of time, the overall cost of a data collector system will be comparable to a low cost surveillance system. In addition to comparable costs, wireless systems provide more frequent readings for better identification of short time-to-failure situations. Cost justifications for many predictive maintenance practices tend to be complex and rely on some intuition, fudge factors, and an experienced practitioner/consultant. A traditional method 
for vibration analysis is to assess the speed degradation to failure and the cost consequence of the machine's failure. This method is well proven, but as technology changes, the justification chart also changes. On-line surveillance systems have become more cost effective, so they have migrated down to the data collector area where there is more overlap of appropriate systems (Figure 1).

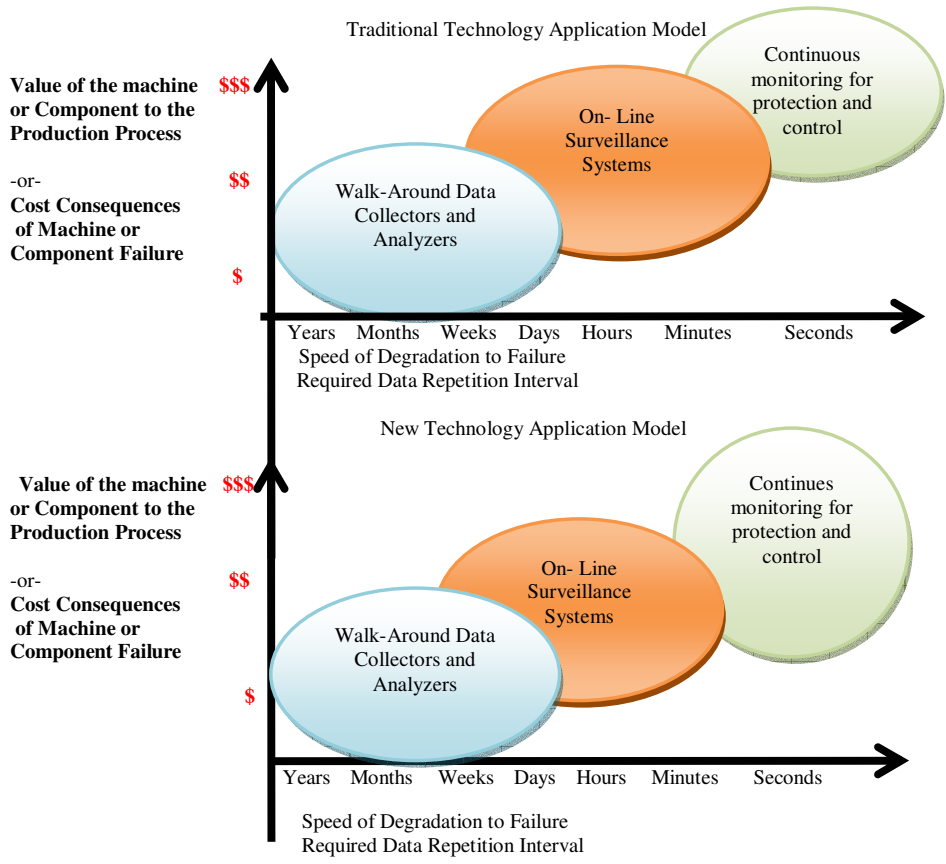

Figure1. The Traditional Technology Application Model evolves as technology reduces the costs of online surveillance systems. Low-cost wireless sensors provide the same cost per point as data collectors while providing more frequent readings [6].

For example, a good candidate for a lower cost surveillance system for a blower motor on top of a furnace or a drive motor protected by heavy guards is hard-wired accelerometers, cables, and a switch box (totaling several hundred dollars). Companies are electing to install on-line surveillance systems on more difficult applications, because these areas are expensive to analyze with data collectors. A low cost wireless accelerometer allows more of these applications to be monitored.

\section{B. Paradigm shift}

These traditional cost justifications only touch upon a portion of the benefits of widespread condition monitoring sensors. Much like cellular phones, low-cost, wireless CM sensors can change the way manufacturing facilities are run. Many companies understand the importance of vibration analysis, but only apply it to critical applications. The most expensive or rapidly declining components are monitored, but these companies still experience unplanned stoppages because the entire facility is not being monitored; a $\$ 100$ motor, for example, can shut down a $\$ 10,000$ / hour production line. Many facilities account for unplanned downtime with excess production capacity. In petrochemical and petroleum refining plants, up to $10 \%$ of the production capacity is required to account for unplanned maintenance. Widespread usage of condition monitoring sensors can monitor up-to-date machine state so that unscheduled downtime can be significantly reduced. Maintenance tasks can be coordinated with production stoppages so that most, if not all, stoppages can be planned and optimized.

\section{Ease of implementation wireless networks sensor for condition monitoring}

All companies are trying to decrease implementation costs. However, capital investment is not the only consideration, but also installation costs, training, documentation, spare parts, and cost of production losses. The key for widespread implementation is ease of installation and ease of maintenance of the system.

Wireless sensors must have:

- Plug-and-play connectivity - Physically installing the sensor, turning it on, and configuring. In the case of a wireless accelerometer, attaching the sensor with a standard 1/4-28 threaded mounting stud and plugging in the battery. The sensors are configured at a main computer, which should take less than a couple minutes per sensor.

\section{- Cause minimal disruption}

- Intelligence to assist with start-up and maintenance - Intelligent sensors and software that assist with start-up and maintenance are also important for ease of use. A wireless sensor should be able to determine a path back to the main computer database without configuration by an operator. System components such as repeaters should work seamlessly without operator intervention.

- $\quad$ Ease of expansion - The next step in widespread implementation is an easy-to-expand system with no discrete input limitations. Companies can purchase and install a handful of sensors on a monthly basis. Installation doesn't require extensive planning or extended shutdown periods. Sensors can also be relocated very easily.

- Maximum reliability - Sensors mounted within range of several transceivers should allow overlapping and be error tolerant. As network conditions change the system should also adjust and adapt so that data collection remains reliable without operator interface.

- Long life - Battery life becomes very important when dealing with large quantities of sensors. Power management is critical to battery life because frequently replacing batteries on hundreds of sensors would be unmanageable. 


\section{CONDITION MONITORING AND CONTROL APPLICATIONS IN OIL AND GAS}

The value of wireless networks is becoming apparent to organizations that have found they need real-time access to knowledge about their plant's environment, processes, and equipment in order to prevent disruption. Spurred by the recent technological advances, the Industrial Revolution is beginning to impact three very distinct monitoring and control applications: oil and gas, cold chain, and machine health monitoring [7].

\section{A. Oil and Gas}

Oil and gas manufacturing is one industry looking toward wireless sensor networks. New wireless mesh networking topologies are one factor that drives new levels of reliability. In a mesh network topology, each mote has at least two parent motes with which it can communicate. Even if an individual link becomes inoperable, a mote still has a communication path available. This redundant routing ensures resiliency in case of offline motes or broken links. Wireless sensor networks also use a combination of Frequency Hopping Spread Spectrum (FHSS) transmission and time synchronization, varying communications in both frequency and in time to sidestep RF interference problems. This technique ensures that alternate paths are available if any signal is blocked due to RF interference. FHSS technology is particularly useful in industrial environments where intermittent RF interference is common. Agile frequency hopping sidesteps interference by utilizing several discrete frequency slices.

Wireless sensor networks provide adaptive monitoring systems in industrial environments with the flexibility and adaptability needed in a plant's monitoring and control strategy. Wireless motes are placed where needed without the need of specialized RF skills or site surveys, while the network handles the rest such as wireless connectivity, routing redundancy, and frequency agility. Additionally, these networks are adaptable to changes in both the configuration of equipment on the plant floor and in the layout of the network itself. If managers add or remove monitoring devices, the network simply reconfigures itself automatically. As wireless networking technology advances, it is also becoming more cost effective. Current wireless sensor networks are designed to ease development and integration with other systems. No customization, integration, or development is required, and there are no wiring or installation costs. Battery powered motes don't require $\mathrm{AC}$ power, which can make wireless networks suitable for locations where power distribution is not designed for additional monitoring equipment. Because motes self-organize into a functioning mesh network, no site survey or wireless expertise is required, and the installer does not have to program or configure the devices. Wireless mesh sensor networks are also designed to deliver long lives with a minimum of ongoing maintenance. Motes can have a lifetime of five to seven years on a single pair of AA batteries and they report on their power status, so operators or managers can tell when battery replacements are due.
The Wireless Sensor Network solution provides remote monitoring capabilities to oil and gas companies to meet this new technology, regulatory and production demands. Example oil and gas remote monitoring applications include:

- $\quad$ Equipment Condition Based Monitoring (CBM)

- Pressure Relief Valve Monitoring (PRV)

- Tank Level Monitoring

- Pipeline Integrity Monitoring

- Geofence Perimeter Security (Pipeline Monitoring)

\section{B. Condition monitoring of different types of pipelines}

This section presents the different types of pipelines that can benefit from the application of the framework. [8][9][10][11][12][13].

\section{1) Sub-sea pipeline}

The ability to monitor flow and pressure build-ups of the multiple phase mixture (oil, gases, brine) through a pipeline in real-time is critical for the safe and efficient transport of fluids. Because pipelines may span many kilometers along the sea bed, normal maintenance procedures at depths of up to 10,000 feet are costly both in time and resources. Failures due to corrosion could cause release of oil and gas, which negatively impacts the environment, causes loss of production, and presents a significant safety hazard. The ability to remotely measure pressure along the entire pipeline is important in the oil and gas industry for the following reasons:

- Flow problems can be identified more quickly to avoid plugging and catastrophic failure.

- Maintenance strategy on production wells and pipelines can scheduled more efficiently and effectively.

- Hydrocarbon delivery can be maximized.

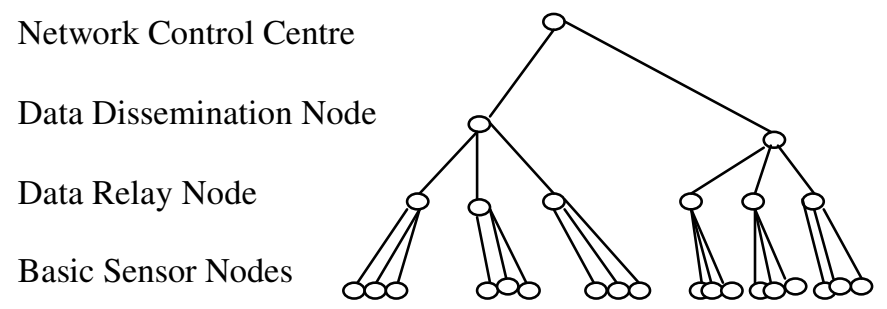

Fig. 2. A hierarchical representation of the linear structure sensor network, showing the parent/child relationship of the various types of nodes.

\section{2) Above ground pipeline}

The temperature of fluid in pipelines will typically differ from that of the atmospheric temperature and will therefore have its own temperature signature which will be 
proportionate to the flow of the fluid. In the event of a leak in the pipeline, the flow downstream of the leak will be less than before the leak, and therefore, there will be different temperature profiles before and after the leak location.

\section{3) Buried pipeline}

In the event of a buried pipeline (typically water pipes are buried) the surrounding areas have large thermal masses and the fluid in the pipeline will approach thermal equilibrium after a certain distance. This means that there will sometimes be a very small temperature change between the fluid in the pipeline and the surrounding environment. This means it is critical that sensors are able to measure very small temperature changes. Typically gas is transported via pipeline in a compressed state. Therefore in the event of a gas leakage this can be particularly dangerous as the stored energy will cause the gas to expand rapidly upon entering the atmosphere. As the gas expands, it also cools rapidly and so there will be a temperature drop at the point of leakage.

\section{4) Gas pipeline}

Typically gas is transported via pipeline in a compressed state. Therefore in the event of a gas leakage this can be particularly dangerous as there is stored energy and the gas will expand rapidly upon entering the atmosphere. As the gas expands it also cools rapidly and so there will be a temperature drop at the point of leakage.

\section{5) Networking model overview and hierarchy}

In this section, the architectural model of the sensor network is presented. Even though an overview of the model is presented, more details about it can be found in [14]. In addition, the routing protocol that is used to collect and route sensor data from the sensing nodes to the data collection, dissemination, and base station nodes is discussed.

In the hierarchical model used, three types of nodes are defined:

Basic Sensor Nodes (BSN): These are the most common nodes in the network. Their function is to perform the sensing function and communicate this information to the data relay nodes.

Data Relay Nodes (DRN): These nodes serve as information collection nodes for the data gathered by the sensor nodes in their one-hop neighborhood. The distance between these nodes is determined by the communication range of the networking MAC protocol used.

Data Discharge Nodes (DDN): These nodes perform the function of discharging the collected data to the Network Control Center (NCC). The technology used to communication the data from these nodes to the NCC center can vary. Satellite cellular technology can be used for example. This implies that each of the DDN nodes would have this communication capability. These nodes are less frequent than the DRN nodes. Each DRN node reports to one DDN node. Figure 2 shows the hierarchical relationship between the various types of nodes in the sensor network. As shown in the figure 2, multiple BSN nodes transmit their data to one DRN node. In turn, several DRN nodes transmit their data to a DDN node. Finally, all DSN nodes transmit their data to the network control centre.

In many industries, especially manufacturing, equipment performance is of critical importance. In many plants, the maintenance of machine condition or operation has traditionally been performed manually. Bearing problems are one of the most common faults in industrial machines, causing unplanned downtime on essential production equipment. Typically, monitoring vibration to detect bearing problems involves manual recordings, or requires expensive systems or services. Another aspect of machine health monitoring, temperature monitoring, detects abnormal or suboptimal machine behavior in order to prevent equipment from further deterioration. Wireless sensor networks continuously collect information about the condition and behavior of machines within plants. Those responsible for equipment benefit from access to expanded monitoring information at greatly reduced costs, allowing them to prevent disruptions, achieve greater uptime results, and meet production goals.

Accelerometers are used to monitor the condition of bearings and rotors in rotating equipment such as motors, fans, pumps and compressors. Defects in the bearing surfaces and unbalanced or misaligned shafts give rise to vibrations in the machine, which ultimately cause machine failure. The frequency of these vibrations is a function of the bearing or shaft construction and its rotational speed, whilst the amplitude is a function of the severity of the problem. By observing the changes in the vibration spectra of a machine, over time, a skilled engineer or increasingly computer software can interpret its condition and, more importantly, give prior warning of imminent failure. This can be used to facilitate a planned maintenance and repair program, avoiding costly down-time. Historically this monitoring practice has been performed using costly hard-wired sensors or infrequent checks by maintenance personnel equipped with hand held monitoring equipment. Now, self-powered wireless condition monitoring sensor systems are able to provide continuous monitoring of critical plant and machinery, with major operating cost benefits. It is a low cost way to install such monitoring and provides an excellent return on investment.

\section{INDUSTRIAL DEPLOYMENTS}

Wireless sensor networks are being primarily piloted in noncritical industrial monitoring applications including predictive maintenance and condition-based monitoring to enable day to- day machinery monitoring and automation of 
data collection In fact, condition-based monitoring often supplements predictive maintenance by acting as an early warning system. Predictive maintenance applications benefit from automation of the traditional manual process for collecting machine condition data and more frequent sampling, while condition-based monitoring applications benefit from more sensing points. General Cable replacement of wired sensors to reduce cost can be found for temperature sensors [15] and vibration sensors [16]. In these deployments, the sensors support predictive maintenance for machinery by sending sensor measurements to a central database that is later analyzed. Specific applications of wireless sensor networks include motor analysis and machine tool performance. Wireless sensor networks enable condition monitoring systems for small electric motors [17] as well as wire replacement for traditional motor vibration monitoring sensors [18]. In addition, wireless sensor networks enable new in-situ motor analysis opportunities previously not possible with wired sensors including agentbased steady-state motor analysis [19] and onboard oil analysis. Applications to machine tool monitoring include temperature measurement sensors for end-mill inserts [20] and vibration-based condition monitoring for tool breakage [21]. In particular, vibrations in machine tools reduce tool life, are a result of dynamic loading, structural element flexibility, cutting conditions, and spindle characteristics, and are often characterized by stability lobes describing regions of "safe" machining with respect to chatter and surface finish [22]. Wireless sensor networks enable new opportunities not possible with wired sensors such as multisensor data fusion methods to estimate tool wear using vibration monitoring of the spindle and/or work piece. In addition, wireless sensing of current, voltage, and acoustic emission signals is also reported [23].

\section{VIBRATION-ENERGY HARVESTING}

Despite its benefits, continuous condition monitoring is currently only carried out on a very small percentage of installed machinery worldwide, due to the cost and reliability limitations of wired or battery-powered technologies. As industry faces continued pressure to reduce its carbon footprint and deliver increased reliability, plant and operations managers are taking advantage of new technologies in wireless sensing solutions for measuring, monitoring, and control.

Nowadays, looking at alternative energies to power their designs are turning to vibration-energy harvesting, which can be successfully integrated with electronic systems, enabling plant operators to deploy reliable wireless sensing systems plant-wide.

In many applications where condition information is required, energy is available in kinetic form. A vibration energy harvester, also called a generator, is a device that converts the vibration of a piece of plant or machinery into useable electrical energy.

New advanced technology has been produced the first commercially available vibration energy harvester for the industrial market segment - the PMG17. The Perpetual micro generator is a practical and robust vibration energy harvester which uses a magnet and coil arrangement to convert mechanical vibration into electrical energy that can be used to power complete wireless sensor systems, eliminating the need for batteries and hard wiring. The energy produced is sufficient to power numerous, modern wireless sensors over a variety of wireless networks.

This technology enables continuous on-line monitoring of plant assets and has been engineered for absolute maximum reliability. Designed to resonate at twice mains frequency $(100$ or $120 \mathrm{~Hz})$, it gives excellent performance on most AC induction or synchronous motor-powered equipment, and can generate up to $50 \mathrm{~mW}$. Output levels are typically enough to power a wireless transmitter sending several Kbytes of data every few minutes, or temperature readings several times a second. The resonant frequency remains stable across a wide temperature range from $-40^{\circ} \mathrm{C}$ to $+85^{\circ} \mathrm{C}$ and the whole package is hermetically sealed against the environment.

Each piece of plant equipment has a unique signature vibration reading and the devices are installed to measure and transmit vibration spectra. Should the vibration reading change (for example as a result of incipient bearing failure) operators would be alerted immediately, assisting predictive maintenance and also preventing unnecessary costly downtime.

The PMG17 is already in use in the Nyhamna gas plant in Norway, powering its networks wireless sensor nodes. Operating faultlessly despite a wide range of temperatures, the sensors monitor the condition of rotating equipment, the main culprit in production shutdowns, reporting temperature and overall vibration every five minutes.

Engineers are able to monitor changes in the vibration frequency, wirelessly, enabling the early identification of potential problems, and perform proactive condition monitoring as part of standard practice. According to Apprion, the cost is up to 70 per cent less than a wired solution and there are none of the associated risks of unnecessary plant shutdowns.

Powering wireless sensor systems using vibration energyharvesting enables low cost installation and maintenance of continuous on-line condition monitoring of plant and machinery. It is these systems and design solutions that allow engineers and operation managers to proactively manage assets, increase plant efficiency and achieves operational excellence. 
[7] Rob Conant, "Wireless sensor networks: Driving The New Industrial Revolution".

[8] A.C. Bruno, R. Schifini, G.S. Khuner, C.H. Barbosa, J.P. Wikswo, and Camerini C.S. New magnetic techniques for inspection and metalloss assessment of oil pipelines. Journal of Magnetism and Magnetic Materials, 226-230(2):2061-2062, May 2001

This paper presented addressing advantages of condition monitoring by wireless network sensors in oil, gas, pipelines, implementation, industrial deployment, and vibration-energy harvesting. In addition, increased reliability is reached by overcoming faulty intermediate node failures, maximizing node battery life, and extending network lifetime. In the future, more detailed analysis of other aspects of the model, including security, will be done. In addition, performance optimizations will be considered for various sensing and monitoring applications which generate different network traffic conditions and varied QoS requirements.

The following issues will be considered for future work:

- Varieties of wireless sensors are commercially available from different vendors. These sensors need to be surveyed so that an appropriate off-theshelf sensor can be chosen for pipelines monitoring.

- While choosing wireless sensor many factors need to be considered such as energy scavenging module, maximum transmission distance, ambient conditions ...etc.

- More investigation is required concerning routing the sensor (slave) information to the sink (master) node.

- Given the developed reliability model, to allocate target reliability to the overall system a top-down approach for reliability allocation can be deployed.

- $\quad$ Fault tree analysis (FTA) may be used to develop system failure mechanisms and the reliability analysis.

- Non-linear structures of pipelines may be considered. This requires revised system design and the reliability modeling.

\section{REFERENCES}

[1] I.Jawhar and J. Wu. Race-free resource allocation for QoS support in wireless networks. Ad Hoc and Sensor Wireless Networks: An International Journal, 1(3):179-206, May 2005.

[2] I. Jawhar and J. Wu. Qos support in tdma-based mobile ad hoc networks. The Journal of Computer Science and Technology (JCST), 20(6):797-910, November 2005.

[3] S. Nelakuditi, Z.-L. Zhang, R. P. Tsang, and D.H.C.Du. Adaptive proportional routing: a localized QoS routing approach. Networking,IEEE/ACM Transactions on, 10(6):790-804, December 2002.

[4] E. Fernandez, I. Jawhar, M. Petrie, and M. VanHilst. Security of Wireless and Portable Device Networks: An Overview.

[5] S. Hadim, J. Al-Jaroodi, and N. Mohamed. Trends in middleware for mobile ad hoc networks. The Journal of Communications, 1(4):1121, July 2006.

[6] Christopher McLean and Dave Wolfe, "How

Wireless Condition Monitoring Sensors Can

Change How Factories Operate," Tenth Annual

Society of Maintenance and Reliability

Conference Proceedings, pp 129-133.
[9] A. Carrillo, E. Gonzalez, A. Rosas, and A. Marquez. New distributed optical sensor for detection and localization of liquid leaks: Part i. experimental studies. Sensors and Actuators A: Physical, 99(3):229-235, June 2002.

[10] M.S. Greenwood and J.A. Bamberger. Ultrasonic sensor to measure the density of a liquid or slurry during pipeline transport. Ultrasonics, 40(18):413-417, May 2002.

[11] S.C. Huang, W.W. Lin, M.T. Tsai, and M.H. Chen. Fiber optic in-line distributed sensor for detection and localization of the pipeline leaks. Sensors and Actuators A: Physical. In Press, Corrected Proof, Available online, November 2006.

[12] F. Rave, L. Zou, X. Bao, L. Chen, R.F. Huang, and H.A. Khoo. Detection of buckling in steel pipeline and column by the distributed brillouin sensor. Optical Fiber Technology, 12(4):305-311, October 2006

[13] C. Verde. Accommodation of multi-leak location in a pipeline. Control Engineering Practice, 13(8):1071-1078, August 2005.

[14] I. Jawhar, N. Mohamed, and K. Shuaib. A framework for pipeline infrastructure monitoring using wireless sensor networks. The Sixth Annual Wireless Telecommunications Symposium (WTS 2007), IEEE Communication Society/ACM Sigmobile, Pomona, California, U.S.A., April 2007.

[15] Kevan, T. (2005). "Upgrading a Steel Mill Wirelessly," Wireless Sensors Magazine, Qestex, Q4 2005. Monitoring for Predictive Maintenance," Wireless Sensors Magazine, Qestex, Q1 2006.

[17] Lu, B.; T.G. Habetler; R.G. Harley; J.A. Gutierrez; and D.B. Durocher (2007). "Energy evaluation goes wireless.” IEEE Industry Applications Magazine (v13, n2), pp. 17-23.

[18] Jagannath, V.M.D. and B. Raman (2007). "WiBeaM: Wireless Bearing Monitoring System." Communication Systems Software and Middleware, COMSWARE 2007, 2nd International Conference, pp. 1-8.

[19] Sundararajan, V.; A. Redfern; W. Watts; and P. Wright (2004). "Distributed Monitoring of SteadyState System Performance Using Wireless Sensor Networks," ASME International Mechanical Engineering Congress, Nov. 2004.

[20] Wright, P.; D. Dornfeld; R. Hillaire; and N. Ota (2006). "Tool Temperature Measurement and its Integration within a Manufacturing System." Transactions of NAMRI/SME, Vol. 34, pp. 63-70.

[21] Sudararajan, V.; A. Redfern; M. Schneider; and P. Wright (2005). "Wireless Sensor Networks for Machinery Monitoring," ASME International Mechanical Engineering Congress and Exposition.

[22] Altintas, Y. and E. Budak (1995). "Analytical prediction of stability lobes in milling," Annals of the CIRP, Vol. 44, No. 1, pp. 357-362.

[23] Ghosh, N.; Y.B. Ravi; A. Patra; S

Mukhopadhyay; S. Paul; A.R. Mohanty; and A.B. Chattopadhyay (2007). "Estimation of tool wear during CNC milling using neural network-based sensor fusion." Mechanical Systems and Signal Processing (v21, n1), pp. 466-479.
[16] Kevan, T. (2006). "Shipboard Machine 